\title{
Dual Effect of Organogermanium Compound THGP on RIG-I-Mediated Viral Sensing and Viral Replication during Influenza a Virus Infection
}

\author{
Sunanda Baidya ${ }^{1,2,+}\left(\mathbb{D}\right.$, Yoko Nishimoto ${ }^{1,2,+}$, Seiichi Sato ${ }^{1,2, *,+} \mathbb{D}$, Yasuhiro Shimada ${ }^{3}$, Nozomi Sakurai ${ }^{1}$, \\ Hirotaka Nonaka ${ }^{1,2}$, Koki Noguchi ${ }^{1,2}$, Mizuki Kido ${ }^{1,2}$, Satoshi Tadano ${ }^{1,2}$, Kozo Ishikawa ${ }^{1}$, Kai Li 1,2, \\ Aoi Okubo ${ }^{1,2}$, Taisho Yamada ${ }^{1,2}$, Yasuko Orba ${ }^{4,5} \mathbb{D}_{\text {, Michihito Sasaki }}^{4} \mathbb{D}_{\text {, Hirofumi Sawa }}{ }^{4,5,6}$, \\ Hiroko Miyamoto ${ }^{7}$, Ayato Takada ${ }^{5,7}$, , Takashi Nakamura ${ }^{3}$ (D) and Akinori Takaoka ${ }^{1,2, *}$
}

\section{check for} updates

Citation: Baidya, S.; Nishimoto, Y.; Sato, S.; Shimada, Y.; Sakurai, N.;

Nonaka, H.; Noguchi, K.; Kido, M.; Tadano, S.; Ishikawa, K.; et al. Dual Effect of Organogermanium Compound THGP on RIG-I-Mediated Viral Sensing and Viral Replication during Influenza a Virus Infection. Viruses 2021, 13, 1674. https:/ / doi.org/10.3390/v13091674

Academic Editor: Daniel R Perez

Received: 16 July 2021

Accepted: 20 August 2021

Published: 24 August 2021

Publisher's Note: MDPI stays neutral with regard to jurisdictional claims in published maps and institutional affiliations.

Copyright: (c) 2021 by the authors. Licensee MDPI, Basel, Switzerland. This article is an open access article distributed under the terms and conditions of the Creative Commons Attribution (CC BY) license (https:/ / creativecommons.org/licenses/by/ $4.0 /)$.
1 Division of Signaling in Cancer and Immunology, Institute for Genetic Medicine, Hokkaido University, Sapporo 060-0815, Japan; sunanda@igm.hokudai.ac.jp (S.B.); wanchiki4100@live.jp (Y.N.); sakurain@igm.hokudai.ac.jp (N.S.); nonaka@igm.hokudai.ac.jp (H.N.); nk810ru@igm.hokudai.ac.jp (K.N.); utapon1202@igm.hokudai.ac.jp (M.K.); s.tadano@frontier.hokudai.ac.jp (S.T.); oup3p.37725126c@gmail.com (K.I.); likai.19870816@163.com (K.L.); aoringo621@igm.hokudai.ac.jp (A.O.); t-yamada@igm.hokudai.ac.jp (T.Y.)

2 Molecular Medical Biochemistry Unit, Biological Chemistry and Engineering Course, Graduate School of Chemical Sciences and Engineering, Hokkaido University, Sapporo 060-0815, Japan

3 Asai Germanium Research Institute Co., Ltd. Suzuranoka, Hakodate 042-0958, Japan; y.shimada@asai-ge.co.jp (Y.S.); nakamura@asai-ge.co.jp (T.N.)

4 Division of Molecular Pathobiology, International Institute for Zoonosis Control, Hokkaido University, Sapporo 001-0020, Japan; orbay@czc.hokudai.ac.jp (Y.O.); m-sasaki@czc.hokudai.ac.jp (M.S.); sawa@czc.hokudai.ac.jp (H.S.)

5 International Collaboration Unit, International Institute for Zoonosis Control, Hokkaido University, Sapporo 001-0020, Japan; atakada@czc.hokudai.ac.jp

6 Global Virus Network, Baltimore, MD 21201, USA

7 Division of Global Epidemiology, International Institute for Zoonosis Control, Hokkaido University, Sapporo 001-0020, Japan; hirom@czc.hokudai.ac.jp

* Correspondence: seisato@igm.hokudai.ac.jp (S.S.); takaoka@igm.hokudai.ac.jp (A.T.)

+ These authors contributed equally to this work.

Abstract: The interaction of viral nucleic acid with protein factors is a crucial process for initiating viral polymerase-mediated viral genome replication while activating pattern recognition receptor (PRR)-mediated innate immune responses. It has previously been reported that a hydrolysate of Ge-132, 3-(trihydroxygermyl) propanoic acid (THGP), shows a modulatory effect on microbial infections, inflammation, and immune responses. However, the detailed mechanism by which THGP can modify these processes during viral infections remained unknown. Here, we show that THGP can specifically downregulate type I interferon (IFN) production in response to stimulation with a cytosolic RNA sensor RIG-I ligand 5'-triphosphate RNA (3pRNA) but not double-stranded RNA, DNA, or lipopolysaccharide. Consistently, treatment with THGP resulted in the dose-dependent suppression of type I IFN induction upon infections with influenza virus (IAV) and vesicular stomatitis virus, which are known to be mainly sensed by RIG-I. Mechanistically, THGP directly binds to the 5'-triphosphate moiety of viral RNA and competes with RIG-I-mediated recognition. Furthermore, we found that THGP can directly counteract the replication of IAV but not EMCV (encephalitismyocarditis virus), by inhibiting the interaction of viral polymerase with RNA genome. Finally, IAV RNA levels were significantly reduced in the lung tissues of THGP-treated mice when compared with untreated mice. These results suggest a possible therapeutic implication of THGP and show direct antiviral action, together with the suppressive activity of innate inflammation.

Keywords: influenza a virus; viral replication; recognition of $5^{\prime}$-triphosphate RNA; antiviral agent; THGP; RIG-I 


\section{Introduction}

The innate immune system is the first line of host defense against invasion by a variety of microbes. The molecular sensor-mediated recognition of invading microbes is the first critical step that initiates the activation of this system. In particular, during viral infections, virus-associated molecular patterns, viral nucleic acids (RNA and DNA), are mainly targeted by pattern recognition receptors (PRRs), including transmembrane-type Toll-like receptors (e.g., TLR3 and TLR9) and cytoplasmic sensors, such as RIG-I (retinoic acid-inducible gene I) and cGAS (cyclic GMP-AMP synthetase) [1-4]. In most cases, such viral sensors activate their downstream signaling to induce types I and III IFN genes, which confer antiviral states to the cell. Among viral nucleic acid sensors, RIG-I and MDA5 are ubiquitously expressed and recognize viral RNAs in the cytoplasmic space. Particularly, RIG-I is a key cytoplasmic PRR for the detection of RNA viruses, such as influenza virus, hepatitis $C$ virus, hepatitis $B$ virus, measles virus, etc., that can be responsible for human infectious diseases [5-8]. RNA carrying 5'-triphosphate modification (3pRNA) and/or short dsRNA is an essential determinant for RIG-I recognition. Ligand-binding to RIGI activates the ATPase activity to change its conformation. Then oligomerized RIG-I interacts with the adaptor protein MAVS/IPS-1 through the CARD domains, leading to the activation of the downstream gene induction programs such as IFNs and proinflammatory cytokines [1,4].

Germanium (Ge) is a trace element, and compounds of Ge are classified into organic and inorganic forms, which are present in certain plants, such as garlic, water-nut, and pearl barley $[9,10]$. Poly-trans-[(2-carboxyethyl) germasesquioxane] (Ge-132) is a watersoluble organogermanium compound that is also known as bis(2-carboxyethylgermanium) sesquioxide, repagermanium, or 2-carboxyethyl-germasesquioxane. Ge-132 is a polymer and hydrolyzes to 3-(trihydroxygermyl)propanoic acid (THGP) monomer spontaneously in the presence of water. There is accumulating evidence that the hydrolytic monomer of Ge132, THGP (Figure S7A for the chemical structure) exhibits diverse biological activities, such as anti-inflammatory, anti-oxidative, anti-melanogenic, pain relief, immunostimulatory, and tumor suppressive effects [11-17]. Due to the low toxicity of THGP, Ge-132 is used as a dietary supplement and in cosmetics in the United States, Europe, and Japan [18-21], and much attention has been paid to this compound, in that it may have a potential therapeutic application in a wide range of fields including cancer, inflammatory diseases, and infectious diseases.

It was previously reported that this compound shows a protective effect in mice infected with a mouse-adapted strain of influenza virus (H2N2) [22], which was based on the THGP effect through IFN- $\gamma$ inducing activity. However, the detailed mechanisms of the immunomodulatory activities of THGP, as well as its effect on innate sensor-mediated immune responses remains to be poorly understood.

In this study, we found that THGP can downregulate RIG-I-mediated signaling via its competitive inhibition of the interaction between RIG-I and its viral RNA ligand. It is likely that THGP specifically targets the $5^{\prime}$-triphosphate portion of the RIG-I ligand, which can also block the binding of IAV RNA polymerase to viral genome, leading to the suppression of viral replication. Thus, our findings suggest that THGP can show direct antiviral action together with a suppressive activity of RIG-I-mediated innate signaling.

\section{Materials and Methods}

\subsection{Cell Culture}

RAW 264.7, HEK293T, A549, and MDCK cells were purchased from ATCC. All cells were maintained in culture medium as recommended by ATCC. To generate MAVS KO A549 cells, we used pX330 vector [23], which was a gift from F. Zhang (Addgene plasmid \#42230, Watertown, MA). The plasmid containing guide RNA against MAVS gene (5'ATTGCGGCAGATATACTTAT-3', Table S1) was transfected, and puromycin-resistant A549 cells were cloned by limiting dilution. The genomic sequences of clones were verified by DNA sequencing, in addition, the absence of MAVS protein was also confirmed by using 
Western blotting. Cells were assayed by using $0.4 \%$ trypan blue uptake (Invitrogen) for measuring cell growth.

\subsection{Viruses, Antibodies, and Reagents}

IAV (A/Puerto Rico/8/1934 H1N1 strain), VSV (New Jersey strain), and EMCV were used as described previously [24]. Antibodies were used as follows: anti-RIG-I (\#3743S; Cell Signaling Technology (CST), Danvers, MA, USA), anti-MDA-5 (\#5321S; Cell Signaling), anti-MAVS (\#3993S; Cell Signaling), anti-pTBK1 (pS172) (D52C2; CST), anti-TBK1 (EP611Y; Abcam, Cambridge, UK), anti-pIRF-3 (pS396) (4D4G; CST), anti-IRF-3 (39371; Active Motif, Carlsbad, CA, USA), anti- $\beta$-actin (AC-15; Sigma, St, Louis, MO, USA), anti-Flag (M2; Sigma), and anti-GST (B-14; Santa Cruz, Dallas, TX, USA). 3pRNA was prepared by using in vitro transcription under the control of the T7 promoter with MEGAscript (Ambion, Austin, TX, USA) as described previously [7]. PolyI:C and LPS derived from E. coli O111:B4 were obtained from GE Healthcare and Invivogen, respectively. HT-DNA, ATP, deoxy-ATP, actinomycin D, and Zanamivir were purchased from Sigma. Adenosine was purchased from Wako. THGP (Asai Germanium Research Institute, Kawasaki, Japan) was prepared as described previously [17]. Biotinylated 3pRNA and Cy5-3pRNA were prepared by using Label IT Nucleic Acid Labeling Kit, Biotin, or Label IT Nucleic Acid Labeling Kit, Cy5 (Mirus, Madison, WI, USA), respectively, according to the product protocol. As for the preparation of dephosphorylated 3pRNA, 3pRNA was treated with alkaline phosphatase (Roche, Basel, Switzerland), according to the product protocol.

\subsection{MAMPs Stimulation and Transfection}

Stimulation with 3pRNA, polyI:C, and HT-DNA were conducted using Lipofectamine 2000 Reagent (Invitrogen, USA) according to the product protocol. The final concentration of 3pRNA, polyI:C, and HT-DNA were $1 \mu \mathrm{g} / \mathrm{mL}, 1 \mu \mathrm{g} / \mathrm{mL}$, and $4 \mu \mathrm{g} / \mathrm{mL}$, respectively. LPS was directly added into the culture solution at a final concentration of $100 \mathrm{ng} / \mathrm{mL}$. To overexpress RIG-I, MAVS, TBK-1, IRF-3 CA, STING, and TRIF, the plasmids were described previously $[7,25,26]$. The cDNA of MDA 5 was amplified by KOD plus polymerase (Toyobo, Osaka, Japan) and subcloned into pIRM-3HA vector. For plasmid transfection, Lipofectamine 2000 reagent (Invitrogen) was used for HEK293T cells transfection.

\subsection{Viral Infection}

In the case of IAV infection, the culture medium was replaced with DMEM without FBS containing $0.0005 \%$ trypsin, and PR8 was added at 1.0 multiplicity of infection (MOI). VSV was infected at an MOI of 0.1. In the case of EMCV, the culture media was changed to DMEM containing no FBS, and the EMCV was infected at an MOI of 0.1. After $1 \mathrm{~h}, \mathrm{FBS}$ was added to the culture medium to a final concentration of $10 \%$. As for the preparation of viral RNA, IAV RNAs including PR8, A/Aichi/2/68 (H3N2), and X31 (H3N2) were purified viruses from egg fluid. EMCV RNA was purified viruses from culture medium of infected IFNAR1 KO cells. SARS-CoV-2 RNA was prepared from culture medium of Vero cells infected as described [24,27].

\subsection{ELISA}

The levels of mouse IFN- $\beta$ protein in culture medium were measured by using the VeriKine-HS Mouse IFN- $\beta$ Serum ELISA kit (PBL; 42410), following the manufacturer's protocol.

\subsection{Plaque-Forming Assay}

RAW264.7 or A549 cells were infected with IAV for $24 \mathrm{~h}$, culture media were collected and serially diluted from 10-1 to 10-6. MDCK cells were infected. After $1 \mathrm{~h}$ infection, cells were overlaid with MEM/BactoAgar/trypsin mixture $(1 \times$ MEM (Gibco, TX, USA), $0.3 \%$ BSA, $0.28 \% \mathrm{NaHCO}_{3}, 1 \times$ MEM Amino Acids Solution (Gibco), $1 \times$ MEM Vitamine Liquid 
(Gibco), $2 \mathrm{mM}$ L-glutamine, $1 \times$ penicillin streptomycin solution (Sigma), $0.0005 \%$ trypsin, $0.8 \%$ BactoAgar). After 2 days, the plaque number was counted.

\section{7. $q R T-P C R$}

RNA was collected by using ISOGEN (Nippon gene, Tokyo Japan) and dissolved in DNase and RNase free water. DNase I (Invitrogen) was added to the extracted RNA and reacted at $25^{\circ} \mathrm{C}$ for $15 \mathrm{~min}$ to degrade the genomic DNA. After the reaction, DNase I was inactivated by adding $1 \mu \mathrm{L}$ of $25 \mathrm{mM}$ EDTA and reacting at $65^{\circ} \mathrm{C}$ for $10 \mathrm{~min}$. Subsequently, RNA was reverse-transcribed using ReverTra Ace qPCR RT Kit (Toyobo) to synthesize cDNA. Quantitative PCR was performed by the intercalator method using SYBR Premix Ex Taq (Takara, Shiga, Japan), quantified by the StepOnePlus Real-Time PCR System (Applied Biosystems) and analyzed by the $\Delta \Delta \mathrm{Ct}$ method. Gapdh was used as an internal standard. Detailed information about the primers used here is shown in Table S2.

\subsection{Immunofluorescence}

RAW264.7 cells seeded on a coverslip (Matsunami Glass, Osaka, Japan) were treated with THGP for $24 \mathrm{~h}$, stimulated with Cy5-3pRNA, and incubated at $37^{\circ} \mathrm{C}$ for $2 \mathrm{~h}$. Cells were fixed with $4 \%$ paraformaldehyde/phosphate buffer (Wako) for $20 \mathrm{~min}$, permeabilized with $0.2 \%$ Triton-X for $10 \mathrm{~min}$ at room temperature, and blocked with $1 \%$ BSA in PBS. The coverslips were incubated for $1 \mathrm{~h}$ in the primary antibody diluted in $1 \%$ BSA in PBS. Following washing with PBS, the coverslips were incubated for $1 \mathrm{~h}$ in appropriate secondary antibodies conjugated with Alexa Fluor 488/594 (Invitrogen) diluted in 1\% BSA in PBS. Subsequently, the coverslips were mounted in Slowfade Gold antifade reagent (Invitrogen) with Hoechst 33342 (Invitrogen). Confocal microscopy was performed with an IX-81S confocal microscope (Olympus, Tokyo, Japan). More than 30 cells in each condition were randomly chosen and representative images are shown in Figures.

\subsection{FACS Analysis}

The cells treated with Cy5-3pRNA in the presence of THGP were suspended in $2 \%$ FBS-PBS and transferred to a tube with a cell strainer cap (Falcon). The fluorescence in the cells was measured with FACS CantoII (BD), and a total of 20,000 cells were counted, and the proportion of cells containing the fluorescent dye was calculated.

\subsection{Recombinant RIG-I Protein}

GST-tagged RIG-I WT were expressed in Sf9 cells according to the manufacturer's instructions for Bac-to-Bac baculovirus expression system (Invitrogen) and purified with Glutathione Sepharose 4B (GE Healthcare). GST-RIG-I protein was eluted with glutathione. The recombinant RIG-I protein was isolated to approximately $>95 \%$ purity as judged by Coomassie brilliant blue staining.

\subsection{In Vitro RNA Pull down Assay}

After the incubation of indicated concentrations of THGP and biotinylated 3pRNA (100 ng) in lysis buffer (20 mM HEPES, $150 \mathrm{mM} \mathrm{NaCl}, 1 \mathrm{mM}$ EDTA, 0.1\% NP-40, $1 \mathrm{mM}$ PMSF) for $30 \mathrm{~min}$ at room temperature, $2 \mu \mathrm{g}$ of GST-RIG-I was added, and the solution was further mixed by inverting at room temperature for $1 \mathrm{~h}$. Next, Dynabeads M-280 Streptavidin (Invitrogen) were added and mixed by inverting for $1 \mathrm{~h}$ at room temperature and washed 3 times with wash buffer (20 mM HEPES, $150 \mathrm{mM} \mathrm{NaCl}, 1 \mathrm{mM}$ EDTA, 0.1\% NP-40). Then, $20 \mu \mathrm{L}$ of $2 \times$ sample buffer was added and then boiled at $100{ }^{\circ} \mathrm{C}$ for $5 \mathrm{~min}$, then the sample was subjected to Western blotting.

\subsection{Binding Assay Using THGP Immobilized Column}

For the preparation of THGP-conjugated beads, THGP was immobilized with TOYOPEARL AF-Epoxy-650 (Tosoh Bioscience, Tokyo, Japan) via ring-cleavage reaction between the epoxy group and amino acid derivative of THGP. The unreacted epoxy group 
was inactivated with mono-ethanolamine. As for binding assay, the beads were pretreated with $0.5 \mathrm{M} \mathrm{NaOH}$ and washed with RNase-free water until the $\mathrm{pH}$ reached 7.0. 3pRNA, polyI:C, or HT-DNA ( $2 \mu \mathrm{g}$ each) were added to a THGP-immobilized column or control beads, the mixture was incubated with gentle rotation at room temperature for $60 \mathrm{~min}$ and centrifuged for $5 \mathrm{~min}$. The beads were washed 3 times with $100 \%$ methanol and eluted with $0.5 \mathrm{M} \mathrm{HCl}$. The concentration of the nucleic acids was measured using a micro ultraviolet visible spectrophotometer Q5000 (Tomy).

\subsection{Luciferase Assay}

As for the measurement of the promoter activity of IFN- $\beta$ and IFN-stimulated response element (ISRE), cells seeded on 24-well plates were transiently cotransfected with luciferase reporter plasmids (100 ng each of pISRE-Luc (Clontech) and p-125Luc (provided by T. Fujita), together with an expression vector or control vector. As for the IAV luciferase activity based mini genome assay for the measurement of IAV polymerase activity, cells seeded on 24-well plates were co-transfected with expression plasmids for WSN-PB2, -PB1, -PA, and -NP (50 ng each); pPolI-NP(0)luc2(0) (2.5 ng) expresses the firefly luciferase gene between the noncoding regions of the WSN-NP gene (provided by Y. Kawaoka), as described previously [28]. As an internal control, $5 \mathrm{ng}$ renilla luciferase reporter plasmid was transfected simultaneously. At $24 \mathrm{~h}$ after transfection, cells were stimulated with the indicated concentrations of THGP for $24 \mathrm{~h}$, and luciferase activity was measured with the Dual-Luciferase Reporter Assay system (Promega) and a photon counter (Centro LB 960; BERTHOLD).

\subsection{RIP Assay}

A549 MAVS KO cells were lysed with buffer A (20 mM HEPES, 150 mM NaCl, $1 \mathrm{mM}$ EDTA, 1\% NP-40, 1 mM PMSF, 1 mM DTT, $1 \mu \mathrm{g} / \mathrm{mL}$ leupeptin, and $100 \mathrm{U} / \mathrm{mL}$ RNaseOUT (Invitrogen) [pH 7.3]), and $20 \mu \mathrm{L}$ of the supernatant was saved as input. Anti-Flag or control IgG was added to cell lysates. After $2 \mathrm{~h}$ incubation with the antibody, Protein-G Dynabeads (Invitrogen) were added, and the solution was further incubated for $1 \mathrm{~h}$ with gentle shaking. Beads were washed three times with a wash buffer (20 mM HEPES, $150 \mathrm{mM}$ $\mathrm{NaCl}, 1 \mathrm{mM}$ EDTA, 0.5\% NP-40 [pH 7.3]). The immunoprecipitated IAV RNA-like RNA of the reporter gene were eluted with Isogen and analyzed by qRT-PCR with specific primers for firefly luciferase (Table S2). The amount of immunoprecipitated RNAs is represented as a percentile of the amount of input RNA (\%input).

\subsection{Mice Experiment}

MAVS KO mice (\#008634) were obtained from Jackson Laboratory. C57BL/6J mice were obtained from Crea Japan. Mice were pretreated with $30 \mu \mathrm{L}$ of THGP (50 or $100 \mathrm{mg} / \mathrm{kg}$ ) by i.n. injection at $3 \mathrm{~h}$ before infection. Mice were infected with IAV (A/Puerto Rico/8/1934 H1N1 strain, $1 \times 10^{5} \mathrm{pfu} /$ mice; i.n.). During the treatment mice were anesthetized with isoflurane (Baxter). At the indicated time after infection, the lung tissues were used for further experiments. These animal experiments were approved by the committee reviews of Hokkaido University (No. 19-0041). For Hematoxylin-Eosin Stain, formalin-fixed paraffinembedded lung tissues were stained with Mayer's hematoxylin (Wako) and 1\% Eosin Y (Wako) following the manufacturer's protocol.

\subsection{6. ${ }^{1} \mathrm{H}-\mathrm{NMR}$}

The ${ }^{1} \mathrm{H}-\mathrm{NMR}$ spectrum of each sample was measured using a Mercury Plus $300 \mathrm{MHz}$ instrument (Agilent Technologies Inc., Little Falls, CA, USA) as described previously [17].

\subsection{Quantification and Statistical Analysis}

Values are shown as mean \pm s.d. Statistical significance between two samples was determined with a Student's t-test. A log-rank test was used to test for differences in survival between control and THGP-treated mice after IAV infection. 


\section{Results}

\subsection{THGP Inhibits RIG-I-Mediated IFN- $\beta$ mRNA Induction}

To first examine whether THGP affects pattern recognition receptor-mediated innate immune signaling pathways, we measured IFN- $\beta$ mRNA induction in a murine macrophage cell line, RAW264.7, pretreated with THGP upon stimulation with 5'-triphosphate RNA (3pRNA), polyI:C, herring testis-DNA (HT-DNA), and lipopolysaccharide (LPS), which are ligands for RIG-I, MDA5, cGAS, and TLR4, respectively (Figure S1A-D). Treatment with THGP inhibited the IFN- $\beta$ mRNA response induced by 3 pRNA but not the other ligands in a dose-dependent manner (Figure S1A-D). We also confirmed that the production of IFN- $\beta$ in response to $3 p R N A$ but not the others is suppressed by THGP at protein levels (Figure 1A-D). In addition, treatment with THGP also suppressed the phosphorylation of both TBK1 and IRF-3 induced by 3pRNA but not the other ligands (Figure 1E-H). On the other hand, stimulation with THGP alone neither induce IFN- $\beta$ mRNA induction nor affect cell growth and viability (Figure S1E-G). These results suggest that THGP has a suppressive effect on 3pRNA-induced activation of RIG-I pathway.

A

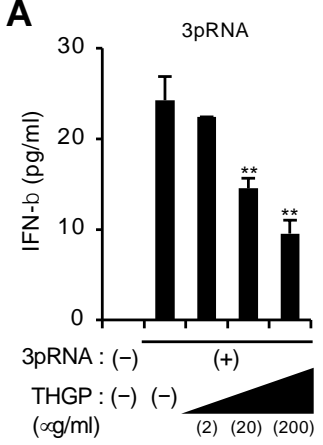

E

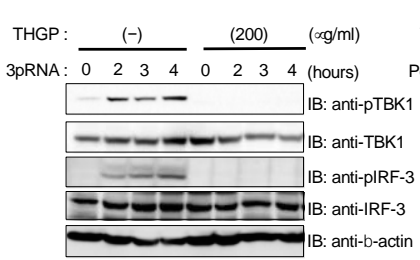

B

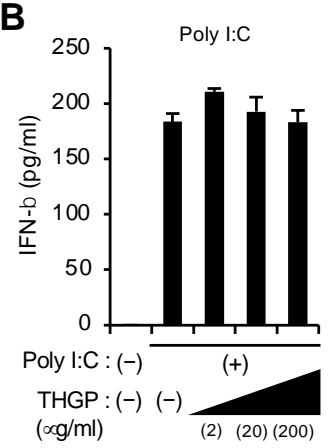

$\mathbf{F}$

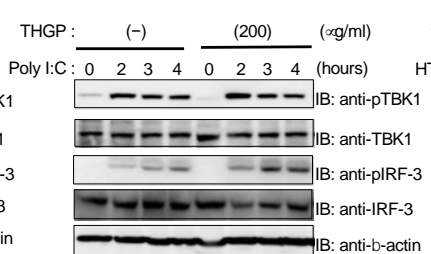

C

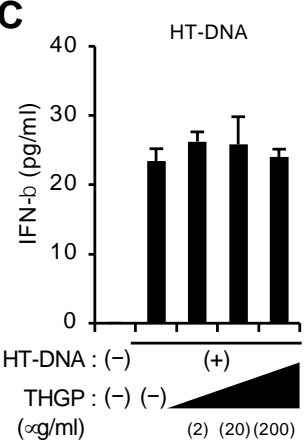

G

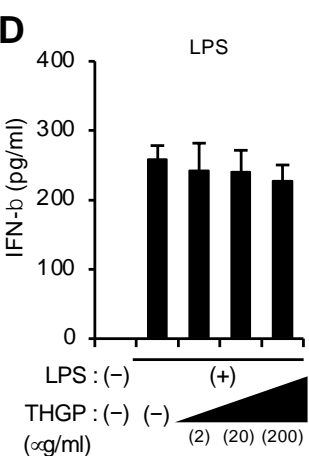

$(\circ \mathrm{g} / \mathrm{ml})$

H

LPS

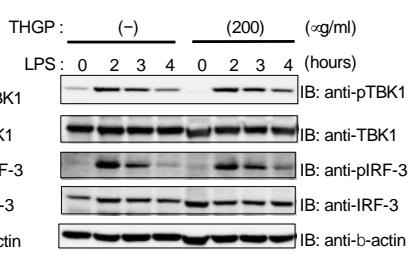

Figure 1. THGP suppresses IFN- $\beta$ induction in response to 3 pRNA but not poly I:C, HT-DNA, and LPS. (A-D) ELISA of IFN- $\beta$ levels at $24 \mathrm{~h}$ after stimulation with 3pRNA (A), polyI:C (B), HT-DNA (C), and LPS (D) in RAW264.7 cells pretreated with indicated concentrations of THGP for $24 \mathrm{~h}$. Data are presented as mean and s.d. $(\mathrm{n}=3)$. (E-H) Whole cell lysates at the indicated time after stimulation with 3pRNA (E), polyI:C (F), HT-DNA (G), and LPS (H) in RAW264.7 cells pretreated with THGP for $24 \mathrm{~h}$, which were prepared and subjected to immunoblotting with anti-pTBK1, TBK1, pIRF-3, IRF-3, and $\beta$-actin antibodies. ${ }^{* *} p<0.01$ vs. control. Data are representative of at least three independent experiments.

\subsection{THGP Attenuates IFN- $\beta$ mRNA Induction in Response to IAV and VSV Infections}

Next, we investigated whether THGP affects the activation of the RIG-I pathway during viral infections. To address this, we tested the effect of THGP on cytokine responses to infections with IAV or vesicular stomatitis (VSV), which are known to be mainly detected by RIG-I. Consistent with the result shown in Figure $1 \mathrm{~A}$, IFN- $\beta$ mRNA induction in response to IAV or VSV infection was suppressed by treatment with THGP in a dosedependent manner (Figure 2A,B), while THGP did not affect IFN- $\beta$ mRNA induction in response to infection with EMCV, which is recognized by MDA5 and does not produces triphosphate RNA [5] (Figure 2C). Similar observation was also obtained for other cytokines induced by innate sensors, such as IL-6, and TNF- $\alpha$ (Figure 2D-I). In addition, we confirmed that THGP reduced the mRNA inductions of IFN- $\beta$ and related genes in response to 3pRNA 
and IAV infection in A549 cells (Figure S2). These results suggest that THGP can suppress cytokine responses downstream of RIG-I but not the MDA5 pathway upon viral infections (Figure S3A).

A

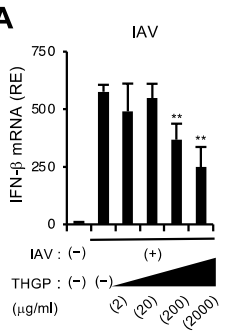

D
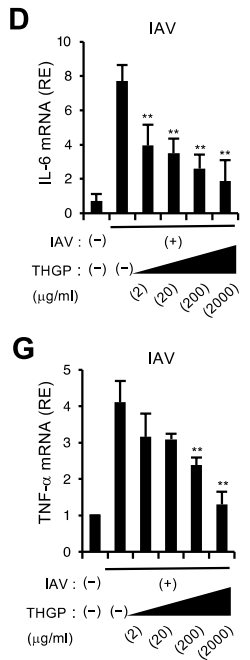

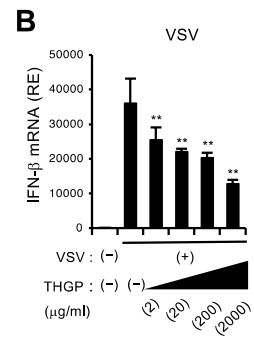

E

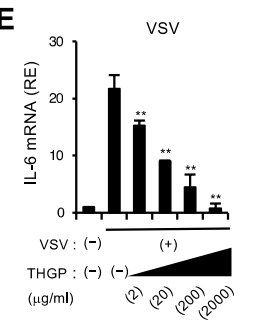

H

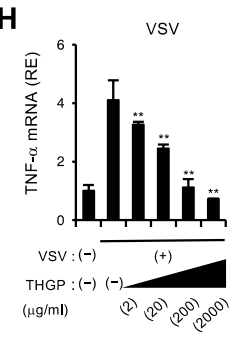

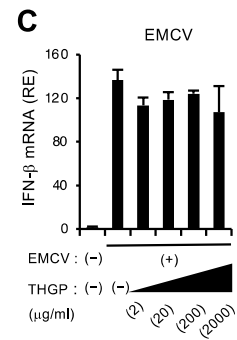

$\mathbf{F}$
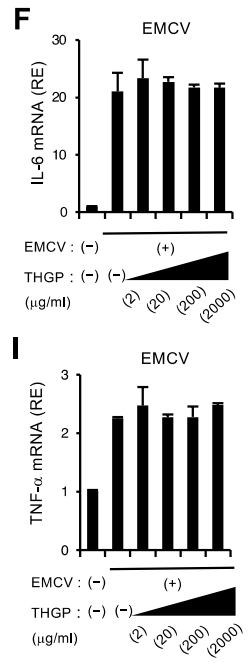

Figure 2. THGP reduces IFN- $\beta$ induction in response to IAV and VSV but not EMCV infections. (A-I) qRT-PCR analysis of IFN- $\beta$ (A-C), IL-6 (D-F), and TNF $\alpha$ (G-I) mRNA levels at $8 \mathrm{~h}$ after infection with control or IAV (A,D,G), VSV (B,E,H), and EMCV $(\mathbf{C}, \mathbf{F}, \mathbf{I})$ in RAW264.7 cells pretreated with the indicated concentrations of THGP for $24 \mathrm{~h} .{ }^{* *} p<0.01$ vs. control. Data are presented as mean and s.d. $(n=3)$ and are representative of at least three independent experiments.

\subsection{THGP Reduces the Interaction between RIG-I and Its Ligand}

To clarify the mechanism for how THGP suppresses the RIG-I pathway, we tried to determine at what level THGP acts on the RIG-I signaling pathway (Figure S3A). We first checked whether THGP affected the activation of the IFN- $\beta$ gene promoter upon the overexpression of RIG-I or its adaptor MAVS. Interestingly, while 3pRNA-induced IFN- $\beta$ gene activation was suppressed by THGP treatment in a dose-dependent manner, it failed to show such a suppressing activity upon RIG-I overexpression even at a high concentration of THGP (Figure 3A,B). In addition, similar results showed that THGP does not affect IFN- $\beta$ gene activation induced by the overexpression of MDA-5, MAVS, STING, TBK1, TRIF, and a constitutively active form of IRF-3 (K152R) (Figure 3C and Figure S3B-F). These results suggest that THGP may act on some process(es) upstream of RIG-I, such as the transfection of 3pRNA into the cytoplasm and/or its interaction with RIG-I. 
A
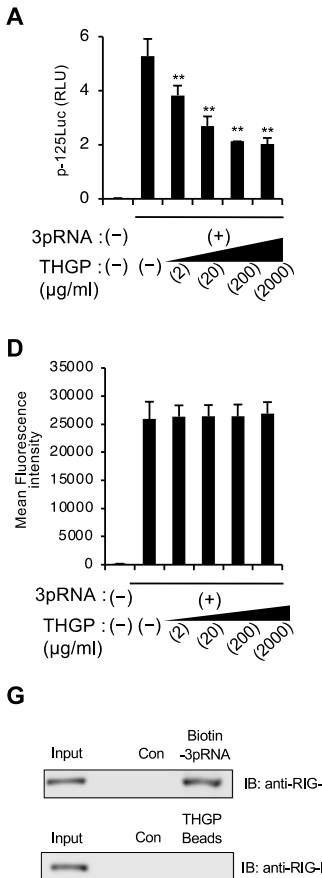

B
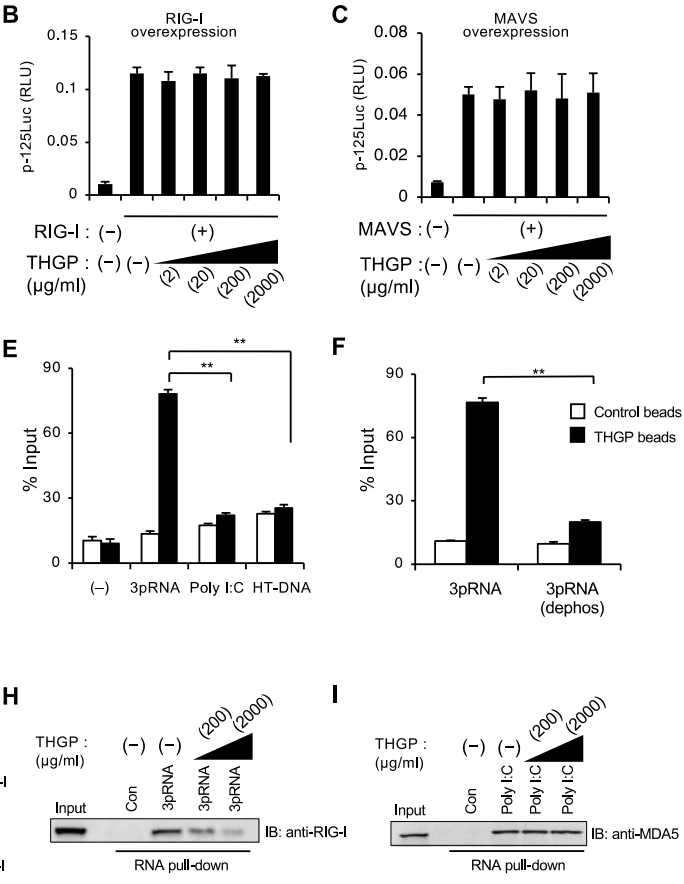

$J$

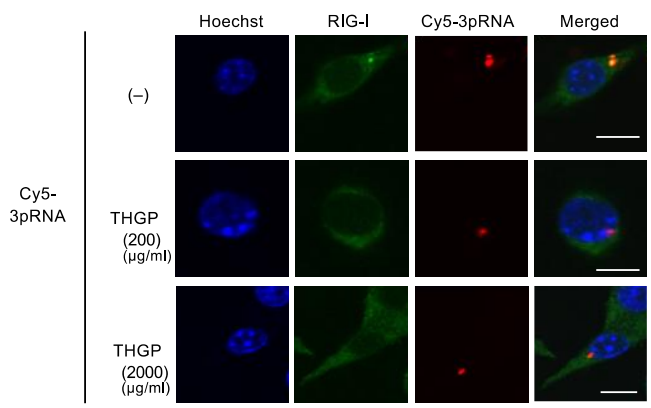

Figure 3. THGP interacts with 3pRNA but not Poly I:C and HT-DNA and inhibits the binding of RIG-I with 3pRNA. $(\mathbf{A}, \mathbf{B})$ Luciferase assay of IFN- $\beta$ gene promoter after treatment of the indicated concentrations of THGP following the transfection of 3pRNA (A), RIG-I (B), and MAVS (C) in HEK293T cells. (D) FACS analysis at $2 \mathrm{~h}$ after transfection with Cy5-3pRNA in RAW264.7 cells. (E) THGP beads pull down assay of 3pRNA, poly I:C, and HT-DNA. The amount of precipitated RNA/DNA with THGP beads (filled bar) or control beads (opened bar) is shown. (F) THGP beads pull down assay of 3pRNA and 3pRNA treated with alkaline phosphatase. The amount of precipitated RNA/DNA with THGP beads (filled bar) or control beads (opened bar) is shown. (G) Pull-down assay showing the binding of biotinylated 3pRNA to RIG-I (top) and THGP to RIG-I (bottom). (H,I) 3pRNA (H) and polyI:C (I) pull down assay, which test the interaction of biotinylated 3pRNA and RIG-I and biotinylated polyI:C and MDA-5 in RAW264.7 cells. Co-precipitated proteins with biotinylated RNA in the presence of indicated concentrations of THGP were subjected to Western blotting using anti-RIG-I and anti-MDA- 5 antibodies. (J) Immunofluorescence analysis for the colocalization of RIG-I and Cy5-3pRNA in the presence of the indicated concentrations of THGP. A representative of more than 30 captured cells is presented. Bar: $10 \mu \mathrm{m} .{ }^{* *} p<0.01 \mathrm{vs.} \mathrm{control.} \mathrm{Data} \mathrm{are}$ presented as mean and s.d. $(n=3)$ and are representative of at least three independent experiments.

We next examined the effect of THGP on the uptake of 3pRNA into the cell. We used Cy5-labelled 3pRNA for transfection into RAW264.7 cells, which were then subjected to FACS and immunofluorescence analyses. However, no effect of THGP treatment on the uptake of Cy5-labeled 3pRNA was observed (Figure 3D and Figure S4A,B). We further tested whether THGP interferes with the interaction between RIG-I and its ligand. Therefore, we prepared THGP-conjugated beads and examined the binding of THGP to RIG-I 
ligands, 3pRNA, and short-form ds-RNA. We found that THGP preferentially associated with 3pRNA but not short-form polyI:C (approximately $4 \mathrm{~kb}$ ) or HT-DNA (Figure 3E). Such an interaction was not observed when we used dephosphorylated 3pRNA, which suggested that its $5^{\prime}$-triphosphate moiety was a key target for the interaction of THGP with 3pRNA (Figure 3F). On the other hand, THGP itself did not show a binding activity to RIG-I protein (Figure 3G). In addition, an RNA pull-down assay with biotin-conjugated 3pRNA showed that endogenous RIG-I was co-precipitated with biotin-conjugated 3pRNA, which was competitively inhibited by THGP treatment (Figure 3H and Figure S4C). On the other hand, THGP did not affect the interaction between MDA5 and polyI:C (Figure 3I). In addition, we observed that the intracellular colocalization of RIG-I with 3pRNA in RAW264.7 cells was suppressed by THGP (Figure 3J). These results suggest that THGP binds to 3pRNA to suppress the interaction between RIG-I and 3pRNA, which leads to the suppressed activation of RIG-I pathway.

\subsection{THGP Impedes the Interaction of IAV Polymerase with Viral RNA Genome}

We next evaluated the effect of THGP treatment on viral genome replication upon infection with IAV, which produces RNA species carrying a $5^{\prime}$-triphosphate moiety that can be a ligand of RIG-I. THGP treatment significantly reduced the viral titers $24 \mathrm{~h}$ after infection without the toxic effect of THGP (Figure 4A and Figure S5A,B). Therefore, we speculated that THGP binding to IAV RNAs might exert a direct antiviral activity on viral replication. In order to test this hypothesis, we used the luciferase activity-based mini genome assay [29] to quantitatively evaluate IAV replication in a human lung cell line, A549. As shown in Figure 4B, we observed that IAV replication was reduced by THGP in a dosedependent manner (Figure 4B). In addition, we used the CRISPR/Cas system to generate MAVS-deficient A549 cells, which failed to induce both RIG-I- and MDA5-mediated innate responses such as type I IFN induction (Figure S5C-F). THGP comparatively also showed a dose-dependent suppressing effect on IAV replication in MAVS-deficient cells (Figure 4C), wherein the activation of the IFN-stimulated response element (ISRE)-driven luciferase gene was not observed (Figure S5G). These results suggest that THGP has direct antiviral activity, possibly through its interaction with the $5^{\prime}$-triphosphate of the IAV genome. The THGP beads pull down assay showed that IAV-derived RNAs, including PR8, Aichi, and X31 but not EMCV and SARS-CoV-2-derived RNA, was remarkably co-precipitated with THGP conjugated with epoxy beads (Figure 4D). In this regard, since it is known that the $5^{\prime}$-region of viral genome contains one of the binding sites of IAV-derived RNA polymerase complex [30], we hypothesized that THGP abrogated the access of IAV polymerase to viral RNA. We used MAVS-deficient A549 cells to remove the possible effect of IFN or IFN-inducible proteins on the interaction between the polymerase and viral RNA-like RNA. As we expected, the RIP assay showed that THGP competitively suppressed the interaction of Flag-tagged polymerase subunit basic protein 2 (PB2) with viral RNA-like RNA in MAVS-deficient A549 cells, in which IAV polymerase subunits, PB1, PA, and NP, as well as Flag-tagged PB2, were reconstituted (Figure 4E). Furthermore, treatment with THGP restored the body weight loss and improved the survival rates of IAV-infected wild-type mice (Figure 4F,G), although the treatment with THGP alone did not show any toxic effect of THGP on survival or the body-weight curves of uninfected mice (Figure S6). Lastly, we used MAVS-deficient mice to assess the effect of THGP on IAV infection in vivo. The viral titers on Day 2 after IAV infection were significantly decreased by THGP treatment (Figure $4 \mathrm{H}$ ). Further, hematoxylin and eosin staining showed that the administration of THGP reduced alveolar hemorrhage, inflammatory infiltration, and interstitial thickening in the alveolar lesions of lung tissue during IAV infection (Figure 4I). Thus, these data suggest that THGP is capable of negatively regulating IAV replication, possibly by blocking the interaction of IAV RNA and viral polymerase. Therefore, THGP functions as a direct antiviral agent that inhibits viral RNA recognition during IAV replication. 
A

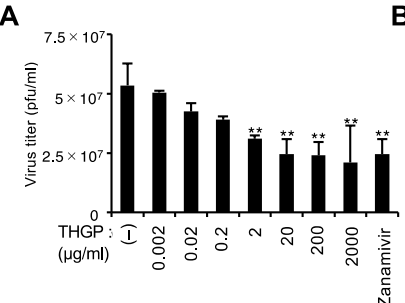

D
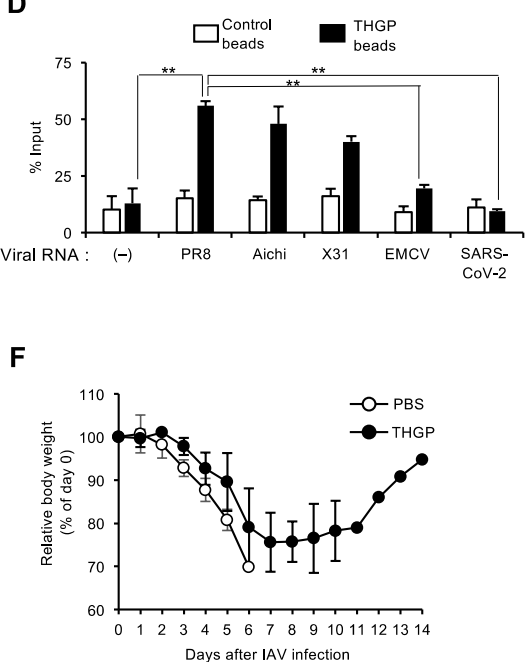

H

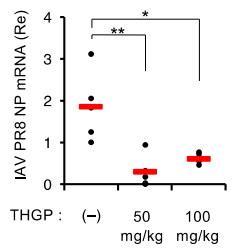

I (-)

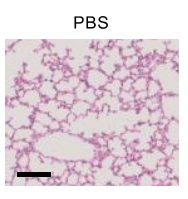

B

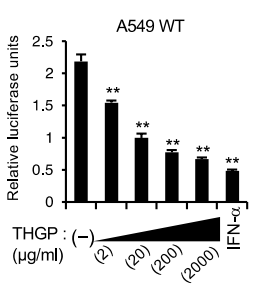

C

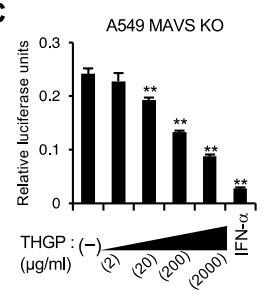

$\mathbf{E}$

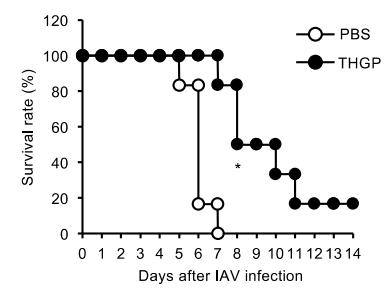

IAV infection
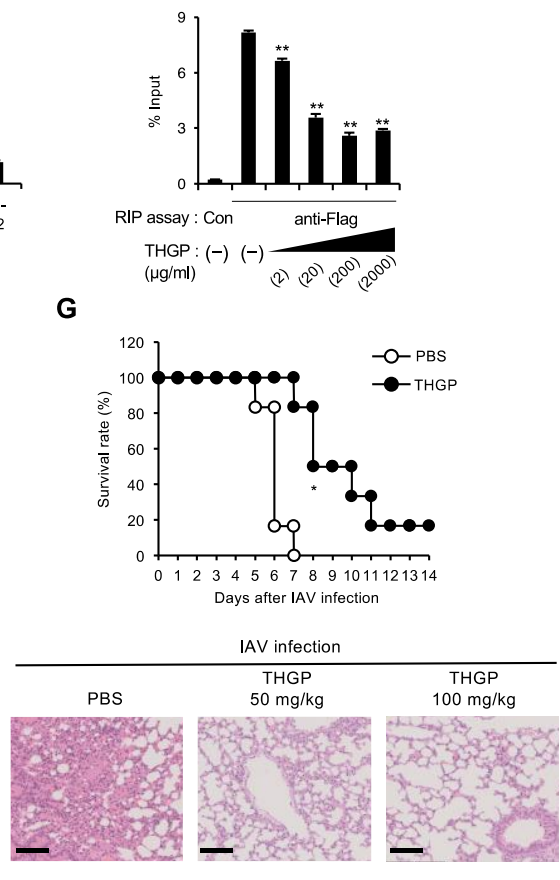

Figure 4. THGP directly abolishes IAV replication in vitro and in vivo. (A) Viral titers measured in MDCK cells after $24 \mathrm{~h}$ of infection with IAV in RAW264.7 cells in the presence of the indicated concentrations of THGP or $0.3 \mathrm{mM}$ zanamivir as control. pfu, plaque-forming units. $\mathrm{n}=3$ samples per group. (B-C) Luciferase activity-based mini-genome assay of IAV replication in the presence of the indicated concentrations of THGP or $1000 \mathrm{U}$ of IFN- $\alpha$ for $24 \mathrm{~h}$ in A549 WT (B) and A549 MAVS KO cells (C) after $24 \mathrm{~h}$ of transfection with IAV-related plasmids, including pPolI/ NP(0)luc2(0), Flag-PB2, PB1, PA, and NP. The $\mathrm{CC}_{50}$ of THGP on A549 cells is evaluated (Figure S5B). (D) THGP pull down assay of viral RNAs including IAV PR8 (A/Puerto Rico/8/1934 H1N1), IAV Aichi (A/Aichi/2/68 H3N2), IAV X31 (H3N2), EMCV, and SARS-CoV-2, which were isolated from purified viruses. The $\%$ input of precipitated viral RNA with THGP beads is shown. (E) RIP assay with A549 MAVS $\mathrm{KO}$ cell lysates prepared after $48 \mathrm{~h}$ of transfection with the IAV-related plasmids including pPolI/ NP(0)luc2(0), Flag-PB2, PB1, PA, NP by using anti-Flag or control immunoglobulin G (con). The immunoprecipitated IAV RNA-like RNA of the reporter gene was measured by qRT-PCR with specific primers for firefly luciferase. (F-G) At $3 \mathrm{~h}$ after intranasal administration with THGP or PBS at a dose of $50 \mathrm{mg} / \mathrm{kg}$ of body weight, C57BL/6J mice were infected intranasally with $10^{5} \mathrm{pfu} /$ animal of IAV PR8. Body weight change (F) and survival rate $(G)$ were monitored ( $n=6$ per group). Mice were intranasally administrated with THGP or PBS at a dose of $50 \mathrm{mg} / \mathrm{kg}$ of body weight every 2 days for 14 days. Day 0 indicates the time of initiation of administration. ${ }^{*} p<0.05$ vs. control (a $\log$ rank-test). We also tested the effect of THGP on the body weight and survival of uninfected WT mice (Figure S6). (H) qRT-PCR analysis of IAV NP RNA levels in the lung tissues of MAVS KO mice at $48 \mathrm{~h}$ post-infection of IAV following administration with PBS (-), THGP (50 mg/kg), or THGP $(100 \mathrm{mg} / \mathrm{kg})$. (I) H\&E stain of lung tissues at 72-h post-infection in MAVS KO mice following i.n. administration with PBS (-), THGP $(50 \mathrm{mg} / \mathrm{kg})$, or THGP $(100 \mathrm{mg} / \mathrm{kg})$. Bar: $100 \mu \mathrm{m} .{ }^{* *} p<0.01 \mathrm{vs}$. control. ${ }^{*} p<0.05$ vs. control. Data are presented as mean and s.d. $(\mathrm{n}=3$ in $(\mathrm{A}-\mathrm{E}) ; \mathrm{n}=5 \mathrm{in}(\mathrm{H}))$ and are representative of at least three independent experiments. 


\section{Discussion}

It has previously been reported that an organo-germanium compound THGP, which is a hydrolysate of Ge-132, shows an antiviral effect on IAV infection. However, the detailed mechanisms of the antiviral activities of THGP, as well as its effect on innate sensor-mediated immune responses were poorly understood. Here, we represented a novel mechanism, by which THGP inhibited not only IAV replication but also RIG-I signaling. For the first time, we demonstrated the suppressing effect of an organogermanium compound, THGP, on the innate signaling pathways activated by RIG-I. In particular, 3pRNA but not ds-RNA-mediated cytokine response was selectively suppressed by THGP treatment. In addition, THGP did not affect other innate signaling mediated by MDA-5, cGAS, and TLR4. Consistent with these results, we also showed that THGP attenuated type I IFN responses and inflammatory cytokines such as IL- 6 and TNF- $\alpha$ upon infections with VSV and IAV, which are reported to be mainly sensed by RIG-I [5,31], whereas such a suppressing effect was not observed upon infection with EMCV, which is recognized by MDA5 [32]. Interestingly, the suppressive effect was not seen by the overexpression of RIG-I and the related signaling proteins such as MDA-5, MAVS, STING, TBK-1, IRF-3, and TRIF. Further investigation revealed that THGP interacted with 3pRNA, which abrogated the RIG-I-mediated recognition of 3pRNA but not the uptake of 3pRNA. According to our data, THGP preferentially interacts with 3pRNA but not polyI:C and HT-DNA. Several reports previously showed that THGP has the characteristic of interacting with compounds containing cis-diol bonds, such as adrenaline, ATP, and L-DOPA [17,19]. Further, an ${ }^{1} \mathrm{H}-\mathrm{NMR}$ analysis comparing ATP and deoxy-ATP here indicated that cis-diol of ATP plays an important role in binding with THGP (Figure S7A-C). In this respect, it was presumable that the cis-diol group of 3pRNA would be a binding site of THGP, but our data indicated that modified 3pRNA, which contain one cis-diol at $3^{\prime}$ end, can still bind to THGP (Figure 3E). The $5^{\prime}$ triphosphate group of 3pRNA was found to be a key portion that was targeted by THGP, which was supported by the result that the dephosphorylation of 3pRNA partially lost the binding activity with THGP (Figure 3F). In addition, our pulldown assay using THGP beads in the presence of competitors, including ATP, deoxy-ATP, or adenosine, suggests that the triphosphate region is more important rather than the cis-diol for the interaction between 3pRNA and THGP (Figure S7D-F). A further detailed $\mathrm{X}$-ray crystallographic analysis is required to structurally show the complex formation between 3pRNA and THGP.

We also showed that THGP interacted with IAV-derived RNA but not EMCV-derived RNA or SARS-CoV-2-derived RNA. The THGP-mediated inhibition of RIG-I signaling led us to test whether THGP promoted viral replication. However, we observed that IAV replication was also suppressed by THGP treatment. Our current data indicated that THGP has a dual effect on the RIG-I-mediated IFN pathway and viral replication, which are in opposition to each other, eventually resulting in decreased viral replication. Mechanistically, we found that THGP binding to IAV RNA restricted the accessibility of viral RNA polymerase to the RNA genome to initiate viral replication without affecting viral RNA stability (Figure 4E, data not shown), suggesting a possible novel direct antiviral action of THGP on IAV RNA or other virus-derived RNAs containing a $5^{\prime}$-triphosphate moiety. It should be noted that viral RNAs of EMCV and SARS-CoV-2, which contains 7-methylguanosine $(\mathrm{m} 7 \mathrm{G})$ cap at the terminal phosphate group of $5^{\prime}$ end [33], did not interact with THGP. Based on the mechanism we found, it is expected that THGP may show antiviral activity against other RNA viruses that contain $5^{\prime}$ triphosphate modification in their genomes, such as vesicular stomatitis virus (VSV) and sendai virus (SenV) $[34,35]$. We think that it is an important future issue to explore the range of viruses that are affected by THGP. Currently, neuraminidase (NA) inhibitors such as oseltamivir and zanamivir, or endonuclease inhibitor such as baloxavir marboxil, are used as antiviral drugs for the treatment of IAV [36]. In this regard, our current data may provide a therapeutic potential of THGP as a novel type of antiviral agent that directly target the IAV genome, interfering with the interaction between the viral RNA and the IAV polymerase. 
On the other hand, given our findings on the suppressive effect of THGP on RIG-I signaling, it might also be a new therapeutic agent for patients with inflammatory diseases due to some types of RIG-I hyperactivation. Tricho-hepato-enteric syndrome (THES), a rare autosomal recessive disorder, is caused by mutations in the TTC37 or SKIV2L gene. The superkiller viralicidic activity 2-like (SKIV2L) RNA helicase, one of causative genes for tricho-hepato-enteric syndrome (THES), also known as syndromic diarrhea (SD) or phenotypic diarrhea (PD), is known to be involved in the formation of an RNA exosome that inhibits ER stress, such as the thapsigargin-induced RIG-I signaling pathway [37]. It has been reported that humans with deficiency in SKIV2L have a type I IFN signature in their peripheral blood [37], suggesting a possible therapeutic application of THGP for the treatment of such inflammatory diseases. We have an interesting observation that THGP exhibits a suppressive effect on IFN response in response to thapsigargin treatment in a mouse macrophage cell line RAW264.7 (Tadano. S, Sato. S, and Takaoka. A; unpublished data), suggesting a possible therapeutic application of THGP for the treatment of such inflammatory diseases.

In addition to this, our data also provide a potential for THGP in excessive inflammation during viral infection (Figure 1, Figure 2 and Figure S2). It has been reported that complications or, ultimately, death arising from viral infections such as severe influenza are often associated with the hyperactivation of a proinflammatory cytokine response, which is known as a "cytokine storm" [38]. THGP, which was shown to have a dual activity on the suppression of the RIG-I pathway and direct antiviral effect, might also be promising in such a clinical setting. Our present study provides a novel aspect of THGP as a potentially attractive clinical option for therapy against infectious diseases caused by not only IAV but also possibly other viruses, as well as inflammatory diseases.

Supplementary Materials: The following are available online at https:/ /www.mdpi.com/article/10 $.3390 / v 13091674 / \mathrm{s} 1$, Figure S1: THGP suppresses IFN- $\beta$ induction induced by 3pRNA but does not induce IFN- $\beta$ itself and affect cell growth, Figure S2: THGP reduces IFN induction in response to the stimulation with 3pRNA and infection with IAV in A549 cells, Figure S3: THGP does not affect IFNB1 promoter activity upon the overexpression of MDA-5, TBK-1, STING, IRF-3, and TRIF, Figure S4: THGP does not influence the uptake of 3pRNA but inhibits the interaction between 3pRNA and RIG-I, Figure S5: Effect of THGP on IAV replication in A549 cells, the establishment of MAVS KO A549 cells and their IFN response to RNA ligands, and the effect of THGP on ISRE response, Figure S6: Effect of THGP on body weight and the survival of uninfected WT mice, Figure S7: Supplemental information for the formation of a complex of 3pRNA and THGP, Table S1: gRNA Sequence of MAVS gene, Table S2: Sequences of primers used for qPCR.

Author Contributions: Conceptualization, S.S. and A.T. (Akinori Takaoka); methodology, S.S., Y.S. and T.N.; validation, S.B., Y.N., S.S., Y.S., N.S., H.N., K.N., M.K., S.T., K.I., K.L. and A.O.; formal analysis, S.B., Y.N. and S.S.; investigation, S.B., Y.N., S.S., Y.S. and N.S.; resources, T.Y., Y.O., M.S., H.S., H.M. and A.T. (Ayato Takada); data curation, S.B., Y.N. and S.S.; writing-original draft preparation, S.S. and A.T. (Akinori Takaoka); writing-review and editing, S.B., Y.N., S.S., N.S., M.K., A.O. and A.T. (Akinori Takaoka); visualization, S.S.; supervision, S.S. and A.T. (Akinori Takaoka); project administration, S.S. and A.T. (Akinori Takaoka); funding acquisition, S.S. and A.T. (Akinori Takaoka). All authors have read and agreed to the published version of the manuscript.

Funding: This work was supported by financial support from Asai Germanium Research Institute Co., Ltd.

Institutional Review Board Statement: Not applicable.

Informed Consent Statement: Not applicable.

Data Availability Statement: The data presented in this study are available on request from the corresponding author.

Acknowledgments: We thank H. Kida for VSV; T. Fujita for the luciferase reporter plasmid p-125Luc, Y. Kawaoka for the plasmids for the IAV polymerase, T. Seya. for the pIRM-3HA-TRIF vector, T. Kubota for the pcDNA3.1(-)IRF-3/5D-FLAG vector, and F. Zhang for the pX330 vector. 
Conflicts of Interest: The authors declare no conflict of interest.

\section{References}

1. Takeuchi, O.; Akira, S. Pattern recognition receptors and inflammation. Cell 2010, 140, 805-820. [CrossRef]

2. McNab, F.; Mayer-Barber, K.; Sher, A.; Wack, A.; O'Garra, A. Type I interferons in infectious disease. Nat. Rev. Immunol. 2015, 15, 87-103. [CrossRef]

3. Chen, K.; Liu, J.; Cao, X. Regulation of type I interferon signaling in immunity and inflammation: A comprehensive review. J. Autoimmun. 2017, 83, 1-11. [CrossRef] [PubMed]

4. Takaoka, A.; Yamada, T. Regulation of signaling mediated by nucleic acid sensors for innate interferon-mediated responses during viral infection. Int. Immunol. 2019, 31, 477-488. [CrossRef] [PubMed]

5. Kato, H.; Takeuchi, O.; Sato, S.; Yoneyama, M.; Yamamoto, M.; Matsui, K.; Uematsu, S.; Jung, A.; Kawai, T.; Ishii, K.J.; et al. Differential roles of MDA5 and RIG-I helicases in the recognition of RNA viruses. Nature 2006, 441, 101-105. [CrossRef] [PubMed]

6. Saito, T.; Owen, D.M.; Jiang, F.; Marcotrigiano, J.; Gale, M. Innate immunity induced by composition-dependent RIG-I recognition of hepatitis C virus RNA. Nature 2008, 454, 523-527. [CrossRef]

7. Sato, S.; Li, K.; Kameyama, T.; Hayashi, T.; Ishida, Y.; Murakami, S.; Watanabe, T.; Iijima, S.; Sakurai, Y.; Watashi, K.; et al. The RNA sensor RIG-I dually functions as an innate sensor and direct antiviral factor for hepatitis B virus. Immunity 2015, 42, 123-132. [CrossRef]

8. Plumet, S.; Herschke, F.; Bourhis, J.M.; Valentin, H.; Longhi, S.; Gerlier, D. Cytosolic 5'-triphosphate ended viral leader transcript of measles virus as activator of the RIG I-mediated interferon response. PLoS ONE 2007, 2, e279. [CrossRef]

9. Kim, E.; Jeon, Y.; Kim, D.Y.; Lee, E.; Hyun, S.H. Antioxidative effect of carboxyethylgermanium sesquioxide (Ge-132) on IVM of porcine oocytes and subsequent embryonic development after parthenogenetic activation and IVF. Theriogenology 2015, 84, 226-236. [CrossRef]

10. Wada, T.; Hanyu, T.; Nozaki, K.; Kataoka, K.; Kawatani, T.; Asahi, T.; Sawamura, N. Antioxidant Activity of Ge-132, a Synthetic Organic Germanium, on Cultured Mammalian Cells. Biol. Pharm. Bull. 2018, 41, 749-753. [CrossRef]

11. Aso, H.; Shibuya, E.; Suzuki, F.; Nakamura, T.; Inoue, H.; Ebina, T.; Ishida, N. Antitumor effect in mice of an organic germanium compound (Ge-132) when different administration methods are used. Gan. Kagaku Ryoho 1985, 12, $2345-2351$.

12. Suzuki, F.; Brutkiewicz, R.R.; Pollard, R.B. Ability of sera from mice treated with Ge-132, an organic germanium compound, to inhibit experimental murine ascites tumours. Br. J. Cancer 1985, 52, 757-763. [CrossRef]

13. Dozono, H.; Ikeda, K.; Onishi, T. Effectiveness of Ge-132 to relieve pain and smooth home care administration for the terminal cancer patient. Gan. Kagaku Ryoho 1996, 23, 291-295.

14. Nakamura, T.; Saito, M.; Aso, H. Effects of a lactobacilli, oligosaccharide and organic germanium intake on the immune responses of mice. Biosci. Biotechnol. Biochem. 2012, 76, 375-377. [CrossRef]

15. Nakamura, T.; Takeda, T.; Tokuji, Y. The Oral Intake of Organic Germanium, Ge-132, Elevates $\alpha$-Tocopherol Levels in the Plas-ma and Modulates Hepatic Gene Expression Profiles to Promote Immune Activation in Mice. Int. J. Vitam. Nutr. Res. 2014, 84, 183-195. [CrossRef] [PubMed]

16. Shimada, Y.; Sato, K.; Takeda, T.; Tokuji, Y. The Organogermanium Compound Ge-132 Interacts with Nucleic Acid Components and Inhibits the Catalysis of Adenosine Substrate by Adenosine Deaminase. Biol. Trace Elem. Res. 2018, 181, 164-172. [CrossRef] [PubMed]

17. Azumi, J.; Takeda, T.; Shimada, Y.; Aso, H.; Nakamura, T. The Organogermanium Compound THGP Suppresses Melanin Synthesis via Complex Formation with L-DOPA on Mushroom Tyrosinase and in B16 4A5 Melanoma Cells. Int. J. Mol. Sci. 2019, 20, 4785. [CrossRef] [PubMed]

18. Sugiya YSakamaki, S.; Sugita, T.; Abo, Y.; Sato, H. Subacute oral toxicity of carboxyethylgermanium sesquioxide (Ge-132) in rats. Ouyou Yakuri 1986, 31, 1181-1190.

19. Nakamura, T.; Shimada, Y.; Takeda, T.; Sato, K.; Akiba, M.; Fukaya, H. Organogermanium compound, Ge-132, forms complexes with adrenaline, ATP and other physiological cis-diol compounds. Future Med. Chem. 2015, 7, 1233-1246. [CrossRef]

20. Doi, Y.; Imai, N.; Suguro, M.; Numano, T.; Furukawa, F. No carcinogenicity of poly-trans-[(2-carboxyethyl) germasesquioxane] (Ge-132): 26-week feeding study using rasH2 mice. Fundam. Toxicol. Sci. 2017, 4, 137-150. [CrossRef]

21. Iwadate, K.; Yamaguchi, Y.; Sasaki, M.; Nakatani, M.; Doi, Y.; Imai, N.; Tamano, S.; Nishihori, Y. Carcinogenicity study of poly-trans-[(2-carboxyethyl)germasesquioxane] (Ge-132) in F344 rats. Fundam. Toxicol. Sci. 2018, 5, 127-140. [CrossRef]

22. Aso, H.; Suzuki, F.; Ebina, T.; Ishida, N. Antiviral activity of carboxyethylgermanium sesquioxide (Ge-132) in mice infected with influenza virus. J. Biol. Response. Mod. 1989, 8, 180-189. [PubMed]

23. Cong, L.; Ran, F.A.; Cox, D.; Lin, S.; Barretto, R.; Habib, N.; Hsu, P.; Wu, X.; Jiang, W.; Marraffini, L.A.; et al. Multiplex genome engineering using CRISPR/Cas systems. Science 2013, 339, 819-823. [CrossRef] [PubMed]

24. Yamada, T.; Horimoto, H.; Kameyama, T.; Hayakawa, S.; Yamato, H.; Dazai, M.; Takada, A.; Kida, H.; Bott, D.; Zhou, A.C.; et al. Constitutive aryl hydrocarbon receptor signaling constrains type I interferon-mediated antiviral innate defense. Nat. Immunol. 2016, 17, 687-694. [CrossRef] [PubMed]

25. Suzuki, H.; Kameyama, T.; Takaoka, A. BinCARD2 as a positive regulator of interferon response in innate immunity. Biochem. Biophys. Res. Commun. 2019, 511, 287-293. [CrossRef] [PubMed] 
26. Sato, S.; Li, K.; Sakurai, N.; Hashizume, M.; Baidya, S.; Nonaka, H.; Noguchi, K.; Ishikawa, K.; Obuse, C.; Takaoka, A. Regulation of an adaptor protein STING by Hsp90ß to enhance innate immune responses against microbial infections. Cell Immunol. 2020, 356, 104188. [CrossRef]

27. Yamada, T.; Sato, S.; Sotoyama, Y.; Orba, Y.; Sawa, H.; Yamauchi, H.; Sasaki, M.; Takaoka, A. RIG-I triggers a signaling-abortive anti-SARS-CoV-2 defense in human lung cells. Nat. Immunol. 2021, 22, 820-828. [CrossRef] [PubMed]

28. Ozawa, M.; Fujii, K.; Muramoto, Y.; Yamada, S.; Yamayoshi, S.; Takada, A.; Goto, H.; Horimoto, T.; Kawaoka, Y. Contributions of two nuclear localization signals of influenza A virus nucleoprotein to viral replication. J. Virol. 2007, 81, 30-41. [CrossRef]

29. Muramoto, Y.; Noda, T.; Kawakami, E.; Akkina, R.; Kawaoka, Y. Identification of novel influenza A virus proteins translated from PA mRNA. J. Virol. 2013, 87, 2455-2462. [CrossRef]

30. Lee, M.T.M. Definition of the minimal viral components required for the initiation of unprimed RNA synthesis by influenza virus RNA polymerase. Nucleic Acids Res. 2002, 30, 429-438. [CrossRef]

31. Yoneyama, M.; Kikuchi, M.; Natsukawa, T.; Shinobu, N.; Imaizumi, T.; Miyagishi, M.; Taira, K.; Akira, S.; Fujita, T. The RNA helicase RIG-I has an essential function in double-stranded RNA-induced innate antiviral responses. Nat. Immunol. 2004, 5, 730-737. [CrossRef] [PubMed]

32. Yoneyama, M.; Kikuchi, M.; Matsumoto, K.; Imaizumi, T.; Miyagishi, M.; Taira, K.; Foy, E.; Loo, Y.-M.; Gale, M., Jr.; Akira, S.; et al. Shared and unique functions of the DExD/H-box helicases RIG-I, MDA5, and LGP2 in antiviral innate immunity. J. Immunol. 2005, 175, 2851-2858. [CrossRef] [PubMed]

33. Romano, M.; Ruggiero, A.; Squeglia, F.; Maga, G.; Berisio, R. A Structural View of SARS-CoV-2 RNA Replication Machinery: RNA Synthesis, Proofreading and Final Capping. Cells 2020, 9, 1267. [CrossRef] [PubMed]

34. Weber, M.; Gawanbacht, A.; Habjan, M.; Rang, A.; Borner, C.; Schmidt, A.M.; Veitinger, S.; Jacob, R.; Devignot, S.; Kochs, G.; et al. Incoming RNA virus nucleocapsids containing a $5^{\prime}$-triphosphorylated genome activate RIG-I and antiviral signaling. Cell Host Microbe 2013, 13, 336-346. [CrossRef] [PubMed]

35. Kolakofsky, D. Isolation and characterization of Sendai virus DI-RNAs. Cell 1976, 8, 547-555. [CrossRef]

36. Dronova, M.; Ikeoka, H.; Itsumura, N.; Hirotsu, N.; Ansaripour, A.; Aballéa, S.; Onishi, Y.; Hill, M.; Igarashi, A. Cost-effectiveness of baloxavir marboxil compared with laninamivir for the treatment of influenza in patients at high risk for complications in Japan. Curr. Med. Res. Opin. 2021, 37, 1135-1148. [CrossRef]

37. Eckard, S.C.; Rice, G.; Fabre, A.; Badens, C.; Gray, E.E.; Hartley, J.L.; Crow, Y.; Stetson, D.B. The SKIV2L RNA exosome limits activation of the RIG-I-like receptors. Nat. Immunol. 2014, 15, 839-845. [CrossRef]

38. Tisoncik, J.R.; Korth, M.J.; Simmons, C.P.; Farrar, J.; Martin, T.R.; Katze, M.G. Into the eye of the cytokine storm. Microbiol. Mol. Biol. Rev. 2012, 76, 16-32. [CrossRef] 